\section{Editorial}

Check for updates

\section{OPEN ACCESS}

Received: Dec 19, 2018

Accepted: Dec 29, 2018

Address for Correspondence:

Hye-Kyung Cho, MD, PhD

Department of Pediatrics, Gachon University

College of Medicine, Gil Medical Center, 21

Namdong-daero 774-beon-gil, Namdong-gu,

Incheon 21565, Republic of Korea.

E-mail: hkcho@gilhospital.com

(c) 2019 The Korean Academy of Medical

Sciences.

This is an Open Access article distributed under the terms of the Creative Commons Attribution Non-Commercial License (https:// creativecommons.org/licenses/by-nc/4.0/) which permits unrestricted non-commercial use, distribution, and reproduction in any medium, provided the original work is properly cited.

ORCID iDs

Hye-Kyung Cho iD

https://orcid.org/0000-0003-0990-1350

Disclosure

The author has no potential conflicts of interest to disclose.

\title{
The Necessity of a Nationwide Surveillance System of Serious BCG Adverse Reactions
}

\section{Hye-Kyung Cho (1)}

Department of Pediatrics, Gachon University College of Medicine, Gil Medical Center, Incheon, Korea

- See the article "Mycobacterium bovis osteitis following immunization with Bacille Calmette-Guérin (BCG) in Korea" in volume 34, e3.

Since the Bacille Calmette-Guérin (BCG) vaccine was first used in 1921, it has been administered safely to billions of people all over the world. Local adverse reactions such as skin reaction and regional lymphadenopathy are common after BCG vaccination, but most of these recover without sequelae. BCG osteitis and generalized BCG infection after BCG vaccination are very rare. The risk of osteitis after BCG vaccination has varied from 0.01 to 300 per million doses, depending on the skill and method of administration, the strain, dose, gender, age, and immune status of the recipient. ${ }^{1}$

In the current issue of Journal of Korean Medical Science, Choi et al. ${ }^{2}$ demonstrated the clinical characteristics of 21 children diagnosed with BCG osteitis during 2007-2018. Although it was reported from a single referral center, it is the first report in Korea that reported a number of cases that occurred over a relatively long period. The clinical features of the patients were comparable to those reported from other countries. ${ }^{3}$ BCG osteitis mainly occurred in children less than 5 years of age and was rarely accompanied by fever $\geq 38^{\circ} \mathrm{C}$. The bones of the limbs, especially lower limbs, were mainly involved rather than the axial bones. Some patients were diagnosed only after a few months after the onset of symptoms, and the authors emphasized the importance of clinical suspicion based on clinical features of BCG osteitis. ${ }^{2}$ There is no national data on the incidence of BCG osteitis in Korea. In their study, the incidence of BCG osteitis following the percutaneous BCG vaccination (Tokyo-172 strain) in Korea was estimated at 4.44 cases per million based on the results of the study. In Taiwan, the isolated extrapulmonary tuberculosis (TB) strains and pathologic specimens collected from children $<5$ years of age have been sent to the national mycobacterial laboratory for detection of BCG strain since 2008. After this active surveillance, the incidence of BCG osteitis increased from 3.7 cases per million doses during 2002-2006 to 30.1 per million during 2008-2012. ${ }^{3}$

In Korea, BCG vaccination is recommended for all newborns within 4 weeks of age, with an immunization rate of up to $99 \%{ }^{4}$; and only intradermal BCG vaccine is included in the national immunization program but percutaneous BCG vaccine is not. Therefore, although the immunization rate of the percutaneous vaccine is more than twice that of the intradermal vaccine, BCG osteitis following percutaneous BCG vaccination has been not reported to the 
vaccination adverse event reporting system. Also, BCG vaccination is being administered to all newborns in Korea without any screening test for severe combined immunodeficiency syndrome (SCID), which is a high-risk condition of disseminated BCG infection. In Taiwan, the timing of BCG vaccination was delayed from 24 hours after birth to 5 months of age in 2012, when SCID screening test is available nationwide, in order to be able to obtain screening results prior to vaccination. In addition, there was a decrease in the TB incidence in Taiwan (48 cases per 100,000 population in 2014), and the schedule of BCG immunization was revised to recommend for infants aged 5-8 months to further decrease the risk of BCG-related severe adverse reactions. ${ }^{5}$ In Japan, the incidence of osteitis after BCG vaccination was reported as two per million doses during 1998-2007.6 In 2013, the recommended age of BCG vaccination was delayed to 5-7 months of age to lower serious complications after the BCG vaccination.

According to the criteria proposed by the International Union against Tuberculosis and Lung Disease, the BCG vaccination policy can be considered to change from routine universal BCG vaccination to selective vaccination of high-risk groups in countries that meet the following conditions. ${ }^{7}$ An efficient notification system should be in place, and one or more of the following three must be met: either the average annual notification rate of smear-positive pulmonary TB is less than five per 100,000 population per year, or the average annual notification rate of TB meningitis in children $<5$ years of age is less than one per 10 million population over the previous 5 years, or the average annual risk of TB infection is less than $0.1 \%$. Under the multifaceted efforts of healthcare providers and public health authorities, the overall incidence of $\mathrm{TB}$ has been gradually decreasing over the past decade in Korea. However, the TB incidence in 2017 is about 70 per 100,000 population, ${ }^{8}$ which is higher than other developed countries and requires routine universal BCG immunization. Therefore, the nationwide incidence of severe adverse reactions following BCG vaccination should be monitored to determine the optimal timing of BCG vaccination.

\section{REFERENCES}

1. Hanekom WA, Hawn TR, Ginsberg AM. Tuberculosis vaccine. In: Plotkin SA, Orestein WA, Offit PA, Edwards KM, editors. Plotkin's Vaccine. Philadelphia, PA: Elsevier; 2018, 1095-113.

2. Choi YY, Han MS, Lee HJ, Yun KW, Shin CH, Yoo WJ, et al. Mycobacterium bovis osteitis following immunization with Bacille Calmette-Guérin (BCG) in Korea. J Korean Med Sci 2019;34(1):e3. CROSSREF

3. Chiu NC, Lin MC, Lin WL, Wang SY, Chi H, Huang LM, et al. Mycobacterium bovis BCG-associated osteomyelitis/osteitis, Taiwan. Emerg Infect Dis 2015;21(3):539-40. PUBMED | CROSSREF

4. Korea Centers for Disease Control and Prevention. National immunization survey in South Korea, 2013. Public Health Wkly Rep 2013;7(21):449-54.

5. Chien YH, Yu HH, Lee NC, Ho HC, Kao SM, Lu MY, et al. Newborn screening for severe combined immunodeficiency in Taiwan. Int J Neonatal Screen 2017;3(16):1-12. CROSSREF

6. Koyama A, Toida I, Nakata S. Osteitis as a complication of BCG vaccination. Kekkaku 2009;84(3):125-32. PUBMED

7. Criteria for discontinuation of vaccination programmes using Bacille Calmette-Guerin (BCG) in countries with a low prevalence of tuberculosis. A statement of the International Union Against Tuberculosis and Lung Disease. Tuber Lung Dis 1994;75(3):179-80. PUBMED | CROSSREF

8. Korea Centers for Disease Control and Prevention. Annual Report on the Notified Tuberculosis in Korea, 2017. Cheongju, Korea: Korea Centers for Disease Control and Prevention; 2018. 